\title{
Ecologie et fonctions des champignons anaérobies du rumen
}

\author{
G. FONTY $\left({ }^{1}\right)\left({ }^{4}\right)$, Elisabeth GRENET $\left({ }^{2}\right)$, M. FEVRE $\left({ }^{3}\right)$, A. BRETON $\left({ }^{1}\right)\left({ }^{5}\right)$, Ph. \\ GOUET $\left({ }^{1}\right)$
}

(1) Laboratoire de Microbiologie, I.N.R.A., Theix, 63122 Ceyrat, France.

${ }^{2}$ ) Station de Recherches sur la Nutrition des Herbivores, Unité Ingestion, I.N.R.A., Theix, 63122 Ceyrat, France.

( $\left.{ }^{3}\right)$ Laboratoire de Différenciation fongique, C.N.R.S. U.A. 1127, Université Lyon, 43, bd du 11-Novembre-1918, 69622 Villeurbanne Cedex, France.

( $\left.{ }^{4}\right)$ Laboratoire de Biologie Comparée des Protistes, C.N.R.S. U.A. 138 , Université Clermont-Ferrand II, 63170 Aubière, France.

$\left({ }^{5}\right)$ Laboratoire de Microbiologie, C.N.R.S. U.A. 045, Université Clermont-Ferrand II 4-6, rue Ledru, 63000 Clermont-Ferrand. France.

Résumé. Les champignons du rumen dont la découverte est récente suscitent un intérêt grandissant depuis quelques années. Leur forte activité cellulolytique "in vitro " et le fait qu'ils colonisent abondamment les tissus ligno-cellulosiques in vivo laissent supposer qu'ils jouent un rôle important dans la dégradation des parois végétales. La présente revue a pour objectif de faire le point des connaissances actuelles sur la biologie de ces micro-organismes. Elle est plus particulièrement focalisée sur les aspects suivants: taxonomie, cycle de développement, exigences nutritionnelles, méthodes de culture, activités métaboliques et équipement enzymatique, colonisation et dégradation des tissus végétaux, influence du régime alimentaire et rôle potentiel in vivo.

Summary. Ecology and functions of rumen anaerobic fungi.

The rumen anaerobic fungi which were discovered recently aroused growing interest over the last few years. Their high cellulolytic activity in vitro, and their ability to heavily colonise the ligno-cellulosic tissues in vivo suggest that they play an important role in plant cell wall degradation. The objective of this paper is to review the current knowledge on the biology of these micro-organisms. It mainly focuses on the following topics: taxonomy, life cycle, nutritional requirements and culture techniques, in vitro metabolic activities and enzymatic equipment, ability to colonise and degrade plant particles in vivo in relation to the diet of the host.

\section{Introduction.}

En 1975, Orpin a découvert que certains micro-organismes flagellés observés depuis longtemps dans le contenu du rumen (Liebetantz, 1910; Braune, 1913) 
et assimilés jusqu'alors à des protozoaires étaient en réalité des zoospores de champignons phycomycètes. L'intérêt de cette identification est devenu évident lorsque les études en microscopie électronique à balayage ont montré que ces champignons colonisaient abondamment les tissus ligno-cellulosiques des particules végétales présentes dans le rumen (Bauchop, 1979a et b).

Cette association étroite avec les fibres végétales suggère qu'ils jouent un rôle dans la dégradation des constituants végétaux. Cette fonction a été confirmée en culture pure (Orpin et Letcher, 1979 ; Bauchop et Mountfort, 1981 ; Akin, Gordon et Hogan, 1983 ; Fonty et al., 1987) et actuellement ces micro-organismes sont étudiés par des équipes britanniques, australiennes, néo-zélandaises, américaines et françaises. Ils ont été mis en évidence chez les bovins, ovins, caprins et cervidés, ainsi que dans le cæcum du cheval, de l'éléphant, et dans l'estomac du kangourou (Bauchop, 1981 ; Orpin, 1981a ; Lowe, Theodorou et Trinci, 1987d).

L'objectif de cette revue est de faire le point des connaissances actuelles sur la biologie et l'écologie de ces micro-organismes en insistant plus particulièrement sur leur rôle dans la dégradation des tissus végétaux.

\section{Cycle de développement et caractéristiques des principales espèces.}

Les espèces actuellement décrites représentent une des formes les plus primitives de fungi (phycomycètes) et possèdent uniquement une reproduction asexuée. Dans leur cycle de développement (fig. 1) alternent des zoospores mobiles libres et une forme végétative non mobile constituée par des sporocystes, pourvus ou non de rhizoïdes, fixés sur les particules alimentaires (Orpin, 1975, $1976,1977 \mathrm{a}, 1984$ ). In vitro, à $39^{\circ} \mathrm{C}$, la durée du cycle varie de 26 à 36 h (Lowe et al., 1987a).

La production de zoospores uni ou pluriflagellées et la présence de chitine dans la paroi ont conduit à classer ces champignons dans les Mastigomycota chytridiomycètes (Orpin, 1975, 1977a). Le genre Neocallimastix prend place dans la nouvelle famille des Neocallimasticaceae, proche des Spizellomycetaceae (Spizellomycetales), créée sur la base des caractères ultrastructuraux de la zoospore et d'une vie strictement anaérobie dans le tube digestif d'animaux vertébrés (Heath et al., 1983). Quatre espèces ont été jusqu'ici décrites et nommées. Une brève clé de détermination permet d'en résumer les caractères essentiels:

a. Zoospores pluriflagellées, sporocystes pourvus de rhizoïdes très ramifiés: Neocallimastix Vavra et Joyon (ex Heath).

a. Zoospores uniflagellées, rarement biflagellées. Sporocystes pourvus de rhizoïdes ramifiés: Piromonas Liebetanz

ou associés à un système de vésicules: Sphaeromonas Liebetanz.

b. Zoospores à 7-10 flagelles et à organelles groupées dans la région flagellaire. Ethanol et formate parmi les produits terminaux de la fermentation: Neocallimastix frontalis (Braune) Vavra et Joyon (ex Heath).

b. Zoospores à 7-17 flagelles et sans distribution bipartite des organelles. Traces 


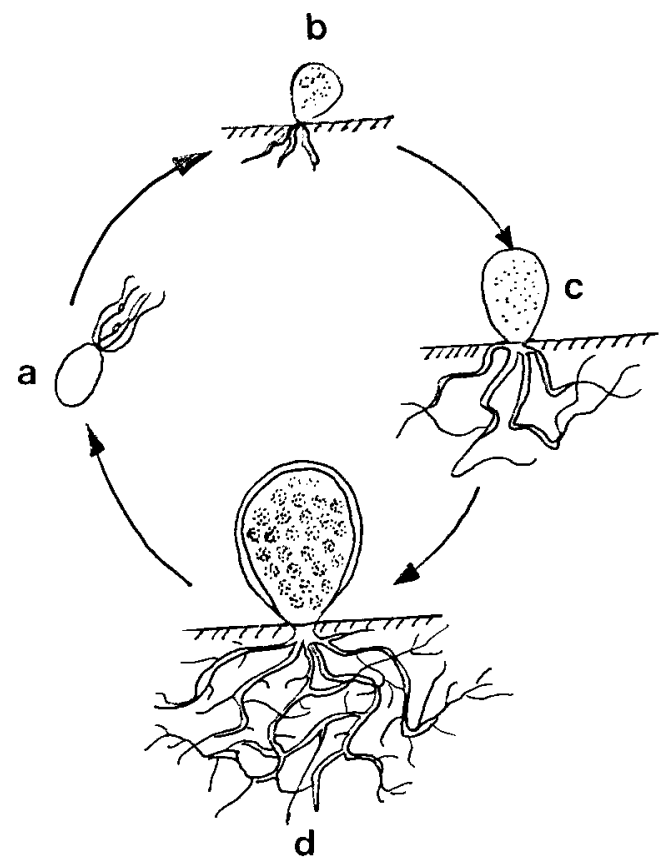

FIG. 1. - Cycle de développement des champignons anaérobies du rumen.

a : zoospore flagellée; $b$ : germination de la zoospore; $c$ et $d$ : différenciation du sporocyste.

d'éthanol et de formate dans les produits terminaux de la fermentation: Neocallimastix patriciarum (Orpin et Munn).

c. Thalle filamenteux: Piromonas communis (Liebetanz).

c. Thalle vésiculeux : Sphaeromonas communis (Liebetanz).

Diverses souches ont encore été décrites sans toutefois être nommées (Lowe et al., 1987a; Ho, Abdullah et Jalaludin, 1988). L'une d'elles, qui se différencie par la formation de structures semblables aux "appressoria » de parasites fongiques mérite probablement le rang d'espèce (Ho, Abdullah et Jalaludin, 1988).

Les zoospores de Neocallimastix frontalis sont uninucléées et renferment diverses inclusions (Munn, Orpin et Hall, 1981). Près du pôle flagellaire sont localisés des organites entourés d'une membrane, des particules, de petites vésicules et des microtubules qui, par groupes de 14 environ, divergent du kinétosome de chaque flagelle. Les organites, présents aussi chez Neocallimastix patriciarum, sont à l'origine d'activités enzymatiques impliquant une hydrogénase et peuvent être en conséquence assimilés à des hydrogénosomes (Yarlett et al., 1986a). II n'y a pas de mitochondries.

Les flagelles qui renferment des doublets de microtubules, disposés en deux rangées concentriques, sont identiques aux flagelles et cils d'autres organismes eucaryotes mobiles. 


\section{Isolement, culture et exigences nutritionnelles.}

Les techniques les mieux adaptées à l'isolement et à la culture des champignons du rumen sont celles préconisées pour les bactéries anaérobies strictes (Hungate, 1969; Aranki et Freter, 1972). Ces micro-organismes sont généralement cultivés sous une atmosphère de $\mathrm{CO}_{2}$ ou d'un mélange $\mathrm{CO}_{2} / \mathrm{N}_{2}$ et incubés à $39^{\circ} \mathrm{C}$, température optimale pour leur développement (Lowe, Theodorou et Trinci, 1987c). Les milieux d'isolement ont une composition proche de ceux utilisés pour les dénombrements bactériens; ils sont rendus sélectifs par l'addition d'un mélange d'antibiotiques (Joblin, 1981; Bauchop, 1979b). L'entretien des cultures nécessite des repiquages tous les 4 à 5 jours mais il semble maintenant possible de conserver des zoospores de Neocallimastix patriciarum plusieurs mois en milieu de culture liquide additionné de $5 \%$ de diméthylsulphoxide puis congelé dans l'azote liquide (Yarlett et al., 1986b).

Les quatre espèces décrites utilisent une large variété de sources carbonées solubles ou insolubles ( $c f .5$ ) et l'azote sous forme d'ammoniaque ou d'acides aminés. Au sein d'une même espèce, les exigences nutritionnelles varient d'une souche à l'autre (Orpin, 1981b) : Neocallimastix a besoin de l'apport de plusieurs porphyrines (hémine, hème, hématine, mésoporphyrine IX, coproporphyrines I et III) (Orpin et Greenwood, 1986a et b) ; la croissance de $N$. patriciarum est stimulée par les acides aminés, les acides acétique, isobutyrique, 2-méthylbutyrique, de faibles concentrations d'acides gras longs et de nombreuses vitamines (Orpin et Greenwood, 1986a). La sporogenèse de $N$. frontalis est induite par la catalase, la peroxidase et la protoporphyrine IX (Orpin et Greenwood, 1986b). Les sulfures sont également indispensables à leur développement (Akin et Hogan, 1983 ; Akin, Gordon et Hogan, 1983 ; Gordon, Gulati et Asher, 1983) et la méthionine est un élément stimulant de leur croissance, tout au moins pour les espèces qui développent un mycélium mais non sur celles de type sphaeromonas (Gordon, 1985). Les exigences de $P$. communis et $S$. communis n'ont pas été déterminées; elles sont néanmoins satisfaites par un apport important (20-40\%) de jus de rumen dans les milieux de culture. Ces espèces peuvent aussi croître sur un milieu semi-défini très complexe sans jus de rumen (Lowe et al., 1985)

\section{Sensibilité des champignons aux antibiotiques et aux acides phéno- liques.}

La croissance de Neocallimastix est inhibée par la nikkomycine (inhibiteur de la chitine synthase) mais non par l'amphotéricine B (ou nystatine) (Lowe et al., 1987a). In vitro, les champignons du rumen sont sensibles aux antibiotiques ionophores mais cette sensibilité varie selon les espèces et les souches (Bernalier et al., 1988; Stewart et al., 1987). Ainsi Sphaeromonas communis est plus sensible que Piromonas et Neocallimastix à la monensine, au lasalocid et à la cationomycine. In vivo, l'effet de la monensine sur la flore fongique est 
controversé; il peut ne diminuer que légèrement la population (E. Grenet, G. Fonty, résultats non publiés), ou au contraire l'éliminer (Elliot et al., 1987). In vitro, les champignons du rumen sont également sensibles aux acides phénoliques. Ainsi, les acides $p$-coumarique, férulique et sinapique à la concentration de $0,1 \%$ inhibent la colonisation et la dégradation des parois végétales par ces micro-organismes (Akin et Rigsby, 1985).

\section{Activités métaboliques in vitro et in vivo et équipement enzymati- que.}

In vitro, les champignons du rumen utilisent une large variété de glucides incluant cellulose (papier filtre), xylane, cellobiose, maltose, saccharose, fructose, xylose, D-mannose, lactose, D-mélibiose (Orpin et Letcher, 1979 ; Fonty et al.. 1987 ; Citron, Breton et Fonty, 1987). La plupart des souches sont par contre incapables de dégrader l'acide polygalacturonique et les pectines. Par un mécanisme de répression catabolique, $N$. frontalis utilise préférentiellement le glucose lorsqu'il est en présence de glucose, fructose et xylose (Mountfort et Asher, 1983). En revanche, aucune régulation n'a été mise en évidence entre maltose, fructose et xylose, ni entre cellobiose et maltose.

Les champignons produisent tous les enzymes nécessaires à la dépolymérisation de la cellulose et des hémicelluloses ainsi qu'à l'hydrolyse des oligosaccharides libérés. Une gamme très vaste d'endoenzymes (cellulases, glucanase, xylanase) et d'exoenzymes ( $\beta$-glucosidase, $\beta$-fructosidase, $\beta$-xylosidase) apparaît dans les cultures de Neocallimastix frontalis en présence de cellulose ou d'hémicelluloses (Williams et Orpin, 1987a, b; Hebraud et Fèvre, 1988a, b). La cellulase de Neocallimastix frontalis cultivé en présence de bactéries méthanogènes a une activité bien supérieure à celle de Trichoderma reesei, qui est un des enzymes les plus actifs isolés jusqu'à présent (Wood et al., 1986, 1987).

Ces enzymes sont principalement exocellulaires: leur production et leur accumulation dans le milieu de culture suivent en parallèle la croissance des champignons (Pearce et Bauchop, 1985; Lowe, Theodorou et Trinci, 1987b; Hebraud et Fèvre, 1988a et b).

Leur synthèse et leur sécrétion sont réprimées par la présence de sucres simples, glucose ou xylose (Mountfort et Asher, 1985 ; Hebraud et Fèvre, 1988a et b) mais elles sont induites par les polymères (cellulose, xylane) ou par des sucres inducteurs comme le cellobiose, le méthylglucoside ou sophorose, caractérisés chez les champignons aérobies (Hebraud et Fèvre, 1988a et b).

Les équipements enzymatiques des différentes espèces de champignons sont identiques. Les enzymes sont produits en quantités comparables lorsque les cultures sont réalisées en présence de substrats complexes (paille, xylane, cellulose) et ils présentent les mêmes caractéristiques de $\mathrm{pH}$, de température et de $\mathrm{Km}$ (Hebraud et Fèvre, 1988a et b). La panoplie des enzymes sécrétés par les champignons ressemble à celles des espèces bactériennes et des protozoaires du rumen (Williams et Strachan, 1984). 
$N$. frontalis fermente la cellulose de papier filtre en produisant formate, acétate, lactate, éthanol, $\mathrm{CO}_{2}$ et $\mathrm{H}_{2}$ (tabl. 1) (Bauchop et Mountfort, 1981 ; Lowe, Theodorou et Trinci, 1987c ; Fonty et al., 1987). Les autres espèces présentent des activités fermentaires similaires; toutefois l'éthanol et le formate ne sont pas produits par $N$. patriciarum (Orpin et Munn, 1986). Toutes les espèces fongiques sont de fortes productrices d'hydrogène. Chez N. patriciarum, cette production est localisée dans des structures intracellulaires appelées hydrogénosomes (Yarlett et al., 1986a) qui ont également été décrites chez des protozoaires du rumen producteurs d' $\mathrm{H}_{2}$ (Yarlett et al., 1981). Cette production d'hydrogène fait intervenir une hydrogénase, la pyruvate ferredoxine oxydo-réductase, une NADPH ferredoxine oxydo-réductase et une enzyme malique. La glycolyse serait la seule voie de fermentation des hexoses chez N. patriciarum (Yarlett et al., 1986a).

TABLEAU 1

Produits terminaux de la fermentation de la cellulose par $\mathrm{N}$. frontalis seul ou associé à Methanobrevibacter sp. (d'après Bauchop et Mountfort, 1981).

\begin{tabular}{lcc}
\hline & \multicolumn{2}{c}{ moles/100 moles hexose } \\
\cline { 2 - 3 } Produits & N. frontalis & $\begin{array}{c}\text { N. frontalis } \\
+ \\
\text { méthanogènes }\end{array}$ \\
\hline Acétate & & 134,7 \\
Lactate & 72,7 & 2,9 \\
Ethanol & 67,0 & 19,0 \\
Méthane & 37,4 & 58,7 \\
Gaz carbonique & 0,0 & 88,7 \\
Hydrogène & 37,6 & 0,05 \\
Formate & 35,3 & 1,0 \\
\hline Carbone retrouvé (\%) & 83,1 & 77,5 \\
$\mathrm{H}_{2}$ retrouvé (\%) & 90,3 & 75,6 \\
O/R & 87,0 & 1,14 \\
Cellulose dégradée (\%) & 1,44 & 82,0 \\
\hline
\end{tabular}

L'absence de cytochrome, de ménaquinone et de mitochondries rend les champignons du rumen complètement dépendants des processus de la fermentation pour la production d'énergie (Mountfort, 1987).

Par leur forte production d'hydrogène ces micro-organismes participent activement à son transfert vers les bactéries hydrogénophiles. Ainsi, en co-culture avec Methanobrevibacter ruminantium le métabolisme de Neocallimastix est dévié vers une plus grande production d'acétate au détriment de la production de lactate et d'éthanol (tabl. 1) (Bauchop et Mountfort, 1981). L'association du champignon avec la bactérie méthanogène se traduit de plus par une augmentation de la vitesse d'hydrolyse et de la quantité de cellulose dégradée. Une 
interaction de même type a également été mise en évidence avec Sphaeromonas et Piromonas (Fonty, Gouet et Santé, 1988). Son mécanisme s'apparente à celui décrit par lanotti et al. (1973) dans le cas du transfert d $\mathrm{d}^{\prime} \mathrm{H}_{2}$ entre Ruminococcus albus et Wolinella succinogenes. In vitro, les champignons interagissent également avec d'autres espèces bactériennes du rumen (Windham et Akin, 1984) et notamment avec Ruminococcus flavefaciens et Selenomonas ruminantium (Bernalier, Fonty et Gouet, 1988).

Les champignons possèdent également une activité protéolytique due à une métalloprotéase inhibée par I'EDTA (Wallace et Joblin). Cette activité pourrait contribuer jusqu'à $30 \%$ à l'activité protéolytique totale du rumen (Wallace et Joblin, 1985 ; Wallace et Munro, 1986). Cette fonction, ajoutée à leur activité cellulolytique, leur procurerait un avantage certain dans la compétition pour la dégradation des parois végétales avec les bactéries cellulolytiques qui, elles, ne possèdent pas d'activité protéolytique.

\section{Fixation des champignons sur les tissus végétaux.}

Les particules alimentaires qui arrivent dans le rumen sont dépourvues de spores de champignons anaérobies (Bauchop, 1979a, 1980). Parmi les composants solubles présents dans les parties aériennes des plantes fourragères, certains induisent la sporogenèse à partir des sporocystes parvenus à maturité et fixés sur les particules antérieurement présentes. On observe ainsi avec les trois espèces fongiques que le nombre de spores augmente immédiatement après le repas (Orpin, 1977c). L'inducteur serait l'hème libre sous sa forme réduite.

Les zoospores ainsi libérées sont attirées par chimiotactisme sur les particules alimentaires nouvellement ingérées et se fixent plus particulièrement sur les tissus endommagés par la mastication (Orpin, 1977c). Quatre chimiorécepteurs ont été mis en évidence (Orpin et Bountiff, 1978) vis-à-vis du glucose, du fructose, du mannose et du mannitol, chacun d'entre eux étant d'ailleurs sensible à plusieurs glucides différents.

Les spores se fixent dans les 15 min qui suivent l'introduction de fragments végétaux et germent pour donner naissance à un mycélium que l'on peut observer $3 \mathrm{~h}$ après leur fixation (Bauchop, 1984). Les rhizoïdes du mycélium pénètrent les tissus végétaux sur une longueur pouvant atteindre 460 microns (Bauchop, 1979b; Ho, Abdullah et Jalaludin, 1988). Les spores se développent lentement pendant $12 \mathrm{~h}$ puis se transforment en sporocystes qui mesurent à maturité, soit après 24 h environ, une centaine de microns (Bauchop, 1979b, 1981 ; Grenet et al., 1988). Elles colonisent une grande variété de tissus et on les trouve plus souvent sur les tiges que sur les feuilles, à moins que ce ne soit des feuilles peu digestibles comme celles de la paille ou du sisal (Agave sisalona) ; le sisal est en effet utilisé dans les cultures de champignons comme substrat solide (Bauchop, 1981). Les spores se fixent sur les tiges de la luzerne et à l'intérieur des tiges des graminées, ou bien, au niveau des stomates (photos 1 et 2) ou sur le sclérenchyme des feuilles de ces dernières (Akin, Gordon et Hogan, 1983 ; Akin, 1987b). 
D’une manière générale, on les trouve sur les tissus à parois épaisses (assise palissadique des pellicules de soja) ou lignifiées (sclérenchyme de la paille, fibres des faisceaux libéro-ligneux de la tige de mais, xylème de la tige de luzerne) (Grenet et Barry, 1988). En revanche, les pulpes de betteraves et les pellicules de graines de colza ne sont que rarement colonisées (Grenet et Barry, 1987)

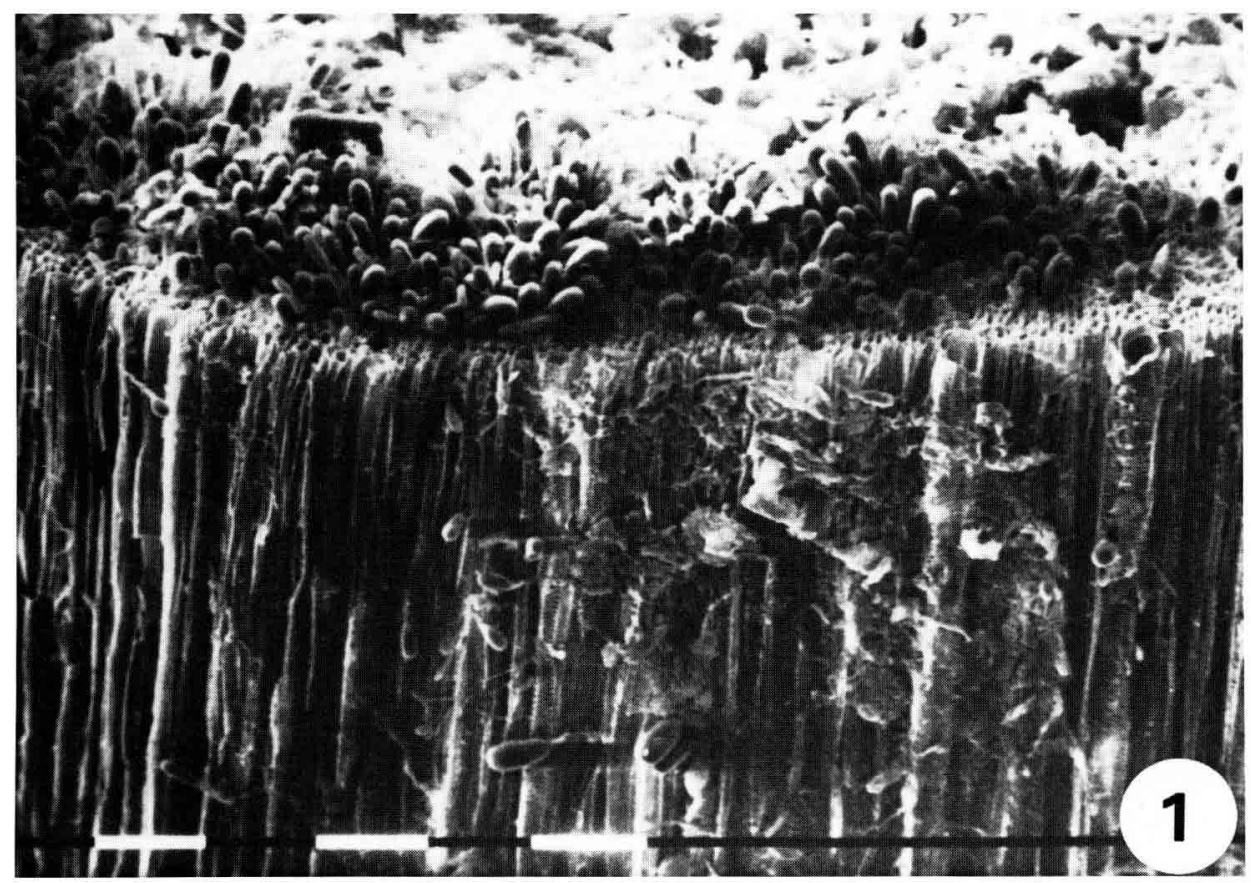

PHOTO 1. - Vue perspective d'une tige de luzerne ayant séjourné $24 \mathrm{~h}$ dans le rumen : le xylène qui a résisté à la dégradation est abondamment colonisé par les champignons anaérobies $(\times 100)$.

\section{Quantification de la biomasse fongique in vivo et influence du régime alimentaire.}

La mesure de la biomasse fongique est indispensable à la quantification de l'activité in vivo des champignons. Alors que les dénombrements bactériens permettent d'estimer la biomasse bactérienne, celui des zoospores ne peut être relié ni à un nombre de sporocystes ni à une quantité de rhizoïdes. Les nombres peuvent varier de 100 fois suivant le régime alimentaire ou le temps après le repas (Orpin, 1975, 1976, 1977c; Fonty et al., 1987). Le dosage de la chitine, constituant spécifique des parois fongiques, est fiable pour des cultures pures (Orpin, 1977b ; Citron, Fonty et Breton, résultats non publiés) mais ne peut être appliqué in vivo. L'extrapolation de résultats obtenus in vitro par cette technique situe la biomasse fongique en moyenne vers $8 \%$ de la biomasse microbienne 


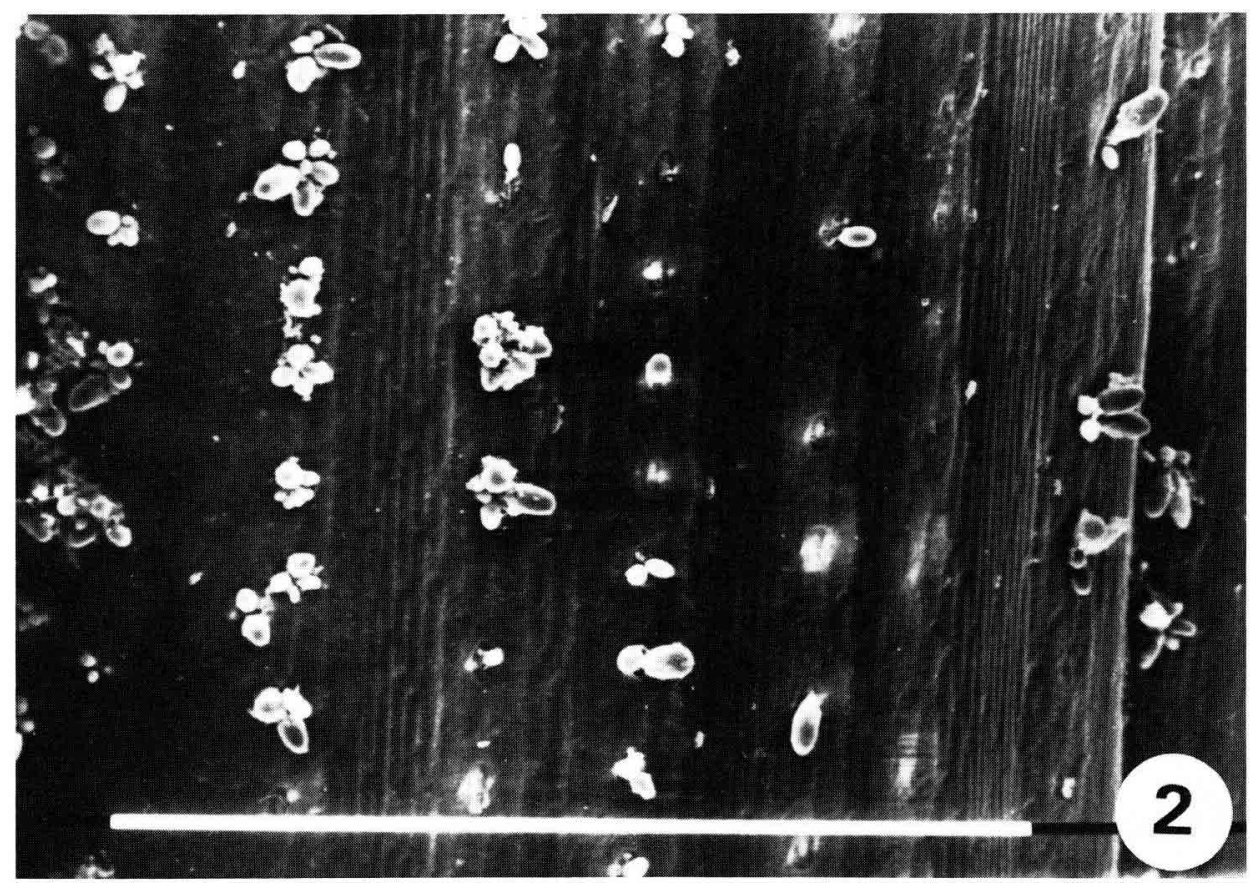

PHOTO 2. - Epiderme d'une feuille de graminée ayant séjourné $24 \mathrm{~h}$ dans le rumen: les champignons anaérobies colonisent les tissus de la feuille au niveau des stomates $(\times 85)$.

totale du rumen (Orpin, 1981b). En solubilisant les parois fongiques par de la chitinase pour des échantillons incubés pendant $48 \mathrm{~h}$, l'estimation serait de $12 \%$ environ du résidu sec du matériel végétal (Akin, 1987a). Le dosage de la chitine synthase présente dans les rhizoïdes pourrait finalement se révéler comme la méthode la plus fiable pour estimer la biomasse in vivo (Gay et al., sous presse).

L'importance de la population fongique estimée par le nombre de spores varie suivant le régime alimentaire (Grenet et al., 1988). Les foins de luzerne ou de prairie naturelle lui sont favorables, contrairement aux fourrages verts pauvres en tiges ou bien à un régime riche en amidon (Bauchop, 1979b, 1981) ou en sucres solubles (Grenet et al., 1988). L'apport de soufre par la fertilisation ou la complémentation de fourrages pauvres (Digitaria pentzii, paille de blé) augmente la population fongique (Gordon, Gulati et Asher, 1983). Des moutons alimentés avec des algues ou recevant de la paille traitée au chlorite de sodium n'hébergeraient aucun champignon (Greenwood et al., 1983 ; Ford, Elliott et Maynard, 1987).

Avec les régimes riches en amidon ou en glucides solubles, qui sont défavorables aux champignons (tabl. 2), le $\mathrm{pH}$ du jus de rumen est généralement inférieur à 5,5. La plus grande concentration en glucides solubles, le taux de renouvellement plus élevé et, par conséquent, le temps de séjour écourté des particules, sont autant d'explications possibles à la diminution de la population 
fongique (Grenet et al., 1989). La régression du nombre de spores s'accompagne d'une colonisation moins abondante des substrats et d'un développement plus réduit des sporocystes. Simultanément, le rapport acide acétique sur acide propionique diminue, conséquence vraisemblable de l'absence des champignons qui sont des micro-organismes acétogènes ne produisant pas d'acide propionique (Ford, Elliott et Maynard, 1987 ; Grenet et al., 1989). Pour un régime donné, les dimensions des sporocystes sont plus importants sur les téguments des graines de soja que sur les tiges de luzerne, les dimensions les plus faibles étant observées sur les tiges de maïs (tabl. 2). Les pulpes de betteraves, quand elles sont colonisées, présentent des sporocystes de petite taille dont on ignore si la croissance a été limitée par la composition du substrat ou s'il s'agit d'espèces différentes.

\section{TABLEAU 2}

Influence du régime alimentaire sur les champignons du rumen; dénombrement des champignons dans le jus de rumen prélevé $2 \mathrm{~h}$ après la distribution du repas chez une vache fistulisée; observation de la colonisation des substrats in sacco et dimensions moyennes (longueur et largeur en $\mathrm{m}$ ) de 10 sporocystes après $24 \mathrm{~h}$ de séjour dans le rumen.

\begin{tabular}{|c|c|c|c|c|c|}
\hline \multirow{2}{*}{ Régime } & \multirow{2}{*}{$\begin{array}{c}\text { Nombre de } \\
\text { zoospores } \\
/ \mathrm{ml} \\
\text { de jus } \\
\left(\times 10^{-3}\right)\end{array}$} & \multicolumn{4}{|c|}{$\begin{array}{l}\text { Colonisation des substrats (a) } \\
\text { Dimensions des sporocystes }\end{array}$} \\
\hline & & $\begin{array}{l}\text { Pulpes de } \\
\text { betteraves }\end{array}$ & $\begin{array}{l}\text { Tiges de } \\
\text { luzerne }\end{array}$ & $\begin{array}{l}\text { Tiges de } \\
\text { mais }\end{array}$ & $\begin{array}{l}\text { Téguments } \\
\text { de soja }\end{array}$ \\
\hline Paille & 20 & $46^{+} \times 43$ & $\begin{array}{l}+++ \\
86 \times 59\end{array}$ & $\begin{array}{c}++ \\
57 \times 54\end{array}$ & $\begin{array}{c}+++ \\
124 \times 64\end{array}$ \\
\hline $\begin{array}{l}\text { Betteraves }(50 \%) \\
+ \\
\text { Foin de luzerne }(50 \%)\end{array}$ & 0,2 & 0 & 0 & 0 & 0 \\
\hline $\begin{array}{l}\text { Orge }(80 \%) \\
\quad+ \\
\text { Ensilage de maîs }(20 \%)\end{array}$ & 0 & 0 & $108^{+} \times 61$ & $66^{+} \times 32$ & 0 \\
\hline
\end{tabular}

(a) : absence de sporocystes; + : sporocystes épais; ++ : sporocystes nombreux $;+++$ : substrats recouverts de sporocystes.

\section{Dégradation des tissus végétaux par les champignons anaérobies.}

Une population mixte de champignons peut dégrader, in vitro, jusqu'à $60 \%$ du matériel végétal mis à incuber (Akin, Gordon et Hogan, 1983), mais l'efficacité à dégrader les polyosides pariétaux varie selon les espèces (Orpin, 1981b, 1984 ; Fonty, Gouet et Santé, 1988 ; Gordon et Ashes, 1984) et selon les souches (Orpin, 1981). Neocallimastix et Piromonas, vraisemblablement à cause de leurs rhizoïdes qui désagrègent les structures végétales, sont plus performants que les espèces non rhizoïdales (Orpin et Hart, 1980; Gordon et Ashes, 1984 ; Orpin, 1984). Une fraction variable de la lignine de la paille de blé, pouvant atteindre 
$20 \%$, est solubilisée dans les cultures de champignons sans que l'on puisse affirmer qu'elle soit utilisée par ces micro-organismes (Orpin, 1981b; Gordon, 1985).

Les observations microscopiques montrent effectivement qu'une partie des tissus lignifiés des feuilles de graminées tropicales est dégradée, en particulier les parois du sclérenchyme (Akin, Gordon et Hogan, 1983 ; Akin et Benner, 1988). Quantifiée au microscope, cette dégradation serait plus active en présence de la flore fongique seule qu'avec l'ensemble de la population microbienne (Akin et Rigsby, 1987).

In vivo, les observations sont peu nombreuses et le rôle des champignons reste encore mal connu. Un régime de paille d'orge délignifiée par le chlorite de soude les élimine et diminue de 4 à $5 \%$ la disparition in sacco de la matière sèche de la paille non traitée (Ford, Elliott et Maynard, 1987) mais, dans ce cas, il est difficile d'établir la part de la disparition de la flore fongique et celle de la modification de la microflore bactérienne consécutive à l'effet du traitement de l'aliment.

Chez les agneaux gnotobiotiques n'hébergeant que Neocallimastix sp. comme seul micro-organisme cellulolytique, $25 \%$ de la cellulose d'une paille de blé et $36 \%$ de celle d'un ray-grass ont disparu in sacco en 48 h (Fonty et Gouet, 1988). Par ailleurs, l'observation microscopique de fragments de tiges de mais

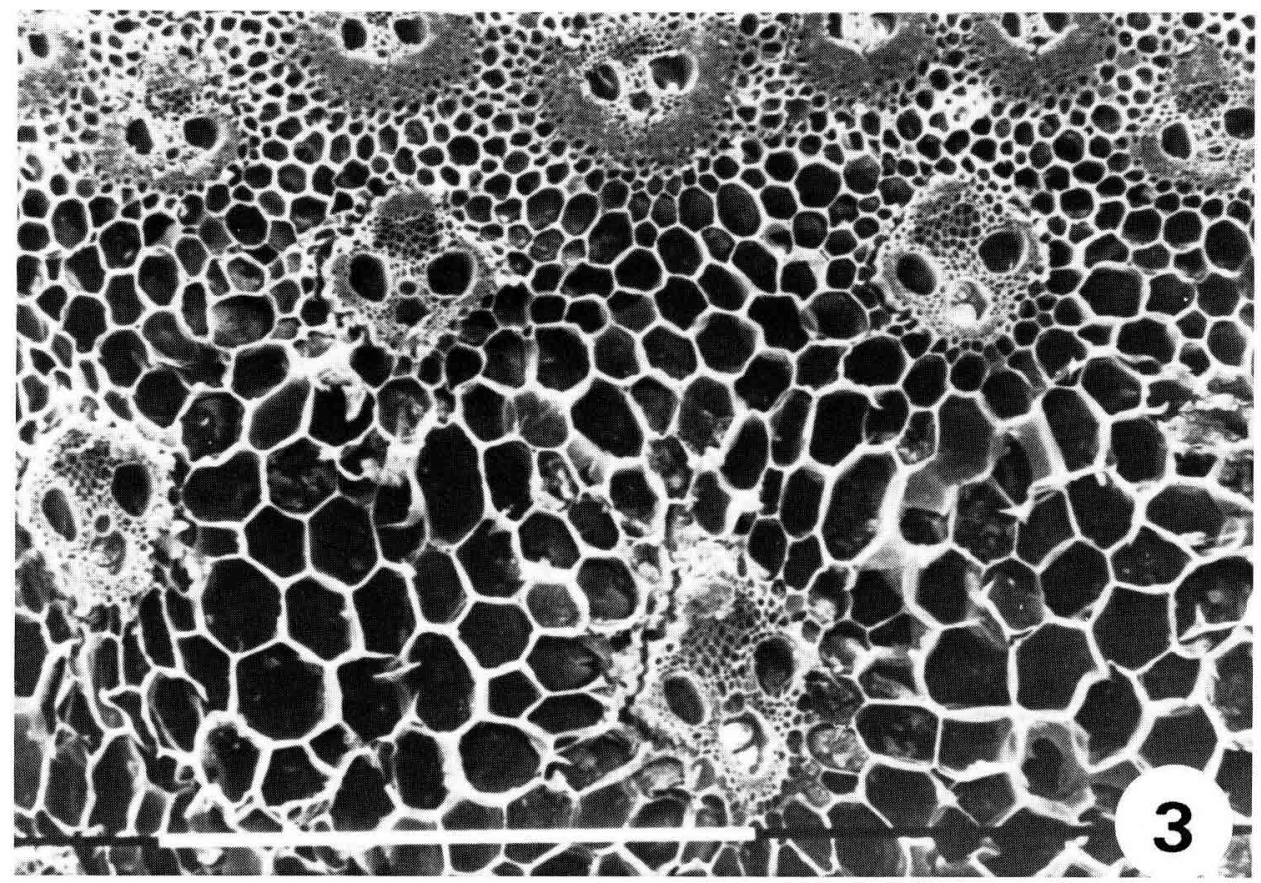

PHOTO 3. - Tige de mais ayant séjourné $8 \mathrm{~h}$ dans le rumen d'un agneau gnotobiotique n'hébergeant que des champignons anaérobies comme micro-organismes cellulolytiques ( $\times 50)$. 
introduits dans des sachets de nylon placés dans le rumen des mêmes agneaux gnotobiotiques montre une dégradation plus lente des tissus que chez un animal " conventionnel » (Grenet, Fonty et Barry, 1988). Les résultats sont néanmoins similaires après $48 \mathrm{~h}$ de séjour ; la colonisation des tissus lignifiés est importante dans le rumen des agneaux gnotobiotiques comme dans le rumen des animaux " conventionnels » mais ce sont les tissus cellulosiques (parenchyme, phloème) qui sont dégradés (photos 3 et 4 ).

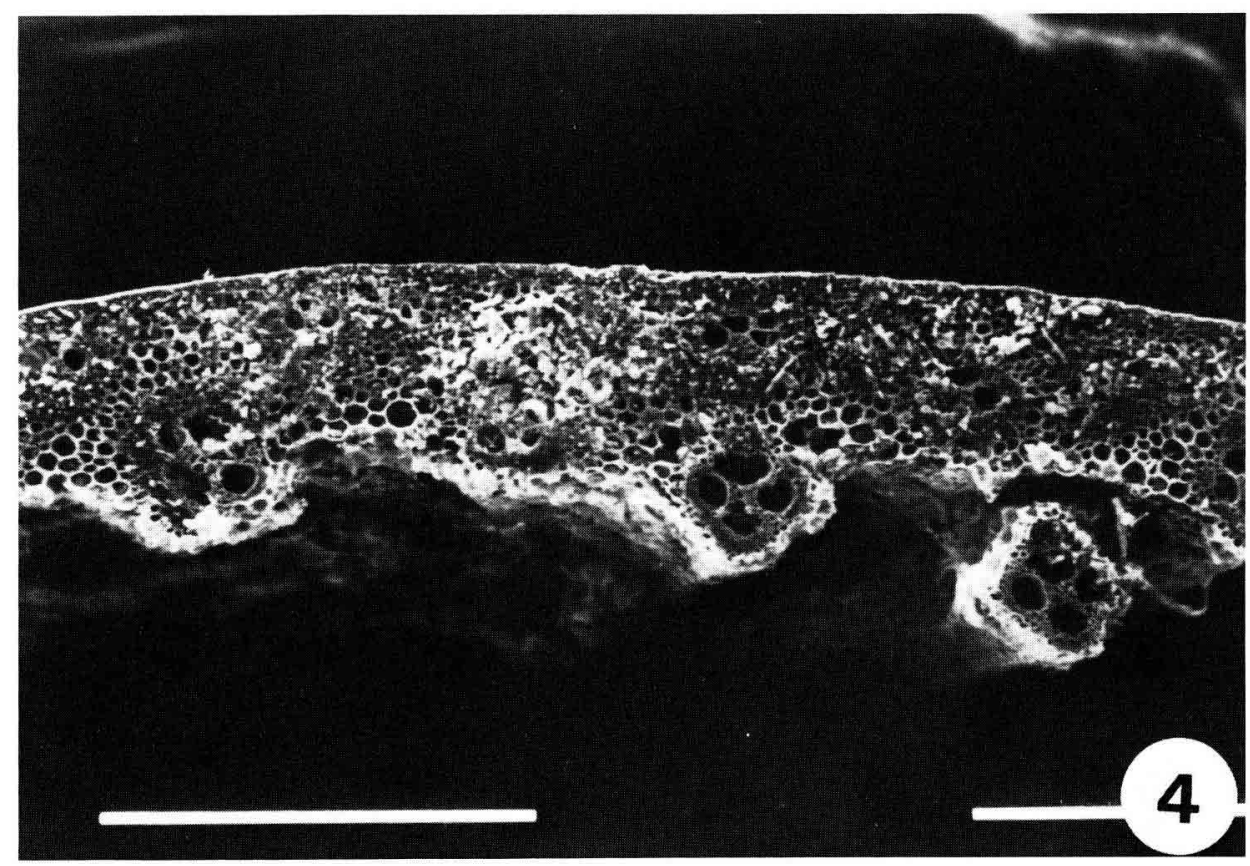

PHOTO 4. - Tige de mä́s ayant séjourné $48 \mathrm{~h}$ dans le rumen d'un agneau gnotobiotique n'hébergeant que des champignons anaérobies comme micro-organismes cellulolytiques: seule la partie la plus externe de la tige, ainsi que le xylème des faisceaux libéro-ligneux, ont résisté $(\times 40)$.

Il semble donc que la présence de champignons soit bénéfique pour l'animal : en accélérant la désagrégation des tissus végétaux ils faciliteraient la colonisation par les bactéries (Orpin, 1984).

\section{Valeur nutritive des champignons pour le ruminant.}

Bien que nous ne possédions que peu d'informations dans ce domaine, il est vraisemblable que les champignons sont utilisés par l'hôte; leur digestibilité in vitro, mesurée par la méthode de Tilley et Terry (1963), se monte à $59 \%$ pour 
Neocallimastix frontalis et $74 \%$ pour Piromonas communis. L'un et l'autre sont riches en matières azotées (44 et $49 \%$ de la matière sèche respectivement) et en protéines ( 24 et $30 \%$ ), une partie de cet azote entrant dans la composition de la chitine formée de molécules de glucosamine (Kemp, Jordan et Orpin, 1985). La composition en acides aminés des protéines de ces deux champignons est comparable à celle de la caséine. Les lipides de Piromonas communis sont composés en majorité de phospholipides (Kemp, Lander et Orpin, 1984).

\section{Conclusion.}

Observés au début du siècle, les champignons anaérobies sont étudiés depuis peu, comme en témoignent les deux tiers des travaux actuellement publiés et cités en référence et qui ont été effectués au cours des 5 dernières années. Malgré cette accélération, les résultats répertoriés reposent encore sur un nombre réduit de travaux dont la plupart devront être confirmés.

$\mathrm{Si}$ la distinction entre 4 espèces majeures (Neocallimastix frontalis, N. patriciarum, Piromonas communis, Sphaeromonas communis) est bien établie, les observations les plus récentes prouvent que parmi les souches actuellement isolées certaines sont totalement différentes et peuvent constituer des espèces nouvelles. Leur inventaire complet et celui des niches écologiques ne sont pas encore terminés. L'étude de leurs cycles, de leur ultrastructure et de leurs besoins nutritionnels doit être approfondie ; la phylogénie pourra progresser rapidement avec les techniques d'hybridation ADN et de séquençage des ARN ribosomaux. Enfin, les milieux de culture et les techniques de conservation gagneront à être perfectionnés.

L'évaluation du rôle de la flore fongique in vivo sur la cellulolyse et la protéolyse reste particulièrement délicate. Si l'utilisation de l'animal gnotobiotique est à même d'entraîner des progrès décisifs, son utilisation en routine n'est pas possible. II est donc indispensable de pouvoir estimer correctement la biomasse fongique et son activité in vivo; le dosage de la chitine synthase, qui donne actuellement de bons résultats in vitro avec des souches pures, pourra peut-être être transféré aux associations microbiennes du rumen.

Les effets des régimes sur la microflore fongique et sa localisation dans le tube digestif, dont les mesures ne présentent aucun problème technique, doivent être poursuivis méthodiquement et systématiquement, aussi bien au plan quantitatif que qualitatif. La mise au point de productions de masse va enfin permettre de déterminer sa composition et sa valeur nutritionnelle.

Les interactions entre la flore fongique et les espèces bactériennes cellulolytiques, les bactéries méthanogènes et d'autres acceptrices $\mathrm{d}^{\prime} \mathrm{H}_{2}$, ainsi que les protozoaires, constituent un vaste champ d'études pour le futur.

Enfin, la purification et la caractérisation des complexes enzymatiques, le clonage des gènes codant pour ces protéines, sont des développements nécessaires pour comprendre le fonctionnement du système lytique des champignons. Ces études pourront alors avoir des conséquences au niveau de l'alimentation des

Reproduction, Nutrition, Développement, 28, Suppl. n¹/88--2 
animaux par l'amélioration des systèmes cellulolytiques, et au niveau biotechnologique par le transfert des gènes à des micro-organismes hypersécréteurs de protéines pour produire en grande quantité des enzymes très actifs.

$4^{\text {mes }}$ Journées sur la Nutrition et l'Alimentation des Herbivores, I.N.R.A., Paris, 24 et 25 mars 1988.

\section{Références}

AKIN D. E., 1987a. Use of chitinase to assess ruminal fungi associated with plant residues in vitro. Appl. environ. Microbiol., 53, 1955-1958.

AKIN D. E., 1987b. Association of rumen fungi with various forage grasses. Anim. Feed Sci. Tech., 16, 273-285.

AKIN D. E., BENNER R., 1988. Degradation of polysaccharides and lignin by ruminal bacteria and fungi. Appl. environ. Microbiol, 54, 1117-1125.

AKIN D. E., HOGAN J. P., 1983. Sulfur fertilization and rumen microbial degradation of cell walls in Digitaria pentzii (Stent). Crop. Sci., 23, $851-858$.

AKIN D. E., GORDON G. L. R., HOGAN J.P., 1983. Rumen bacterial and fungal degradation of Digitaria pentzii grown with and without sulfur. App/ environ. Microbiol., 46, 738-748.

AKIN D. E., RIGSBY L. L., 1985. Influence of phenolic acids on rumen fungi. Agron. J., 77 , 180-182.

AKIN D. E., RIGSBY L. L., 1987. Mixed fungal populations and lignocellulosic tissues degradation in the bovine rumen. Appl. Environ. Microbiol., 53, 1987-1995.

ARANKI A., FRETER R., 1972. Use of an anaerobic glove boxe for the cultivations of strictly anaerobic bacteria. Amer. J. clin. Nutr., 25, 1329-1334.

BAUCHOP T., 1979a. The rumen anaerobic fungi : colonizers of plant fibre. Ann. Rech. Vét., 10, 246-248.

BAUCHOP T., 1979b. Rumen anaerobic fungi of cattle and sheep. Appl. environ. Microbiol., 38 , $148-158$.

BAUCHOP T., 1980. Scanning electron microscopy in the study of microbial digestion of plant fragments in the gut, 309-326. In D. C. ELLWOOD, J. N. EDGER, M. J. LATHANS, M. J. LYNCH, J. H. SLATER, Contemporary microbial ecology. Acad. Press, London.

BAUCHOP T., 1981. The anaerobic fungi in rumen fibre digestion. Agric. Environ., 6, 339-348.

BAUCHOP T., 1984. Rumen anaerobic fungi and the utilization of fibrous feeds. Rev. Rural Sci., 6 , $118-123$.

BAUCHOP T., MOUNTFORT D. O., 1981. Cellulose fermentation by a rumen anaerobic fungus in both the absence and the presence of rumen methanogens. Appl. environ. Microbiol., 42, $1103-1110$.

BERNALIER A., BOGAERT C., FONTY G., JOUANY J. P., 1988. Effect of inophore antibiotics on anaerobic fungi. In J. V. NOLAN, R. A. LENG, D. I. DEMEYER, The role of protozoa and fungi in ruminant digestion. Penambule Books, Armidale N.S.W. 2351. Australia. Proc. Int. Seminar sponsored by the O.E.C.D. Cooperative Research Project on Food and Preservation, and the University of New England, held 26-29, September 1988 (sous presse).

BERNALIER A., FONTY G., GOUET Ph., 1988. Dégradation et fermentation de la cellulose par Neocallimastix sp. $\mathrm{MCH}_{3}$ seul ou associé à quelques espèces bactériennes du rumen. Reprod. Nutr. Dévelop., 28, Suppl. $\mathrm{n}^{\circ} 1$.

BRAUNE R., 1913. Untersuchungen über die im wiederkauermagen vorkommenden Protozoen. Arch. Protistenk, 32, 111-170.

CITRON A., BRETON A., FONTY G., 1987. The rumen anaerobic fungi. Bull. Inst. Pasteur, 85, 329-343.

ELLIOTT R., ASH A.J., CALDERON-CORTES F., NORTON B.W., BAUCHOP T., 1987. The influence of anaerobic fungi on rumen volatile fatty acid concentrations in vivo. J. agr. Sci. Camb., 109, 13-17. 
FONTY G., BRETON A., FEVRE H., CITRON A., HEBRAUD M., GOUET Ph., 1987. Isolement et caractérisation des champignons anaérobies stricts du rumen de moutons. Premiers résultats. Reprod. Nutr. Dévelop., 27, 107-108.

FONTY G., GOUET Ph., 1988. Establishment of microbial populations in the rumen. Utilization of an animal model to study the role of the different cellulolytic micro-organisms in vivo. In J. V. NOLAN, R. A. LENG, D. I. DEMEYER, The role of protozoa and fungi in ruminant digestion. Penambule Books, Armidale N.S.W. 2351, Australia. Proc. Int. Seminar sponsored by the O.E.C.D. Cooperative Research Project on Food and Preservation, and the University of New England, held 26-29, September 1988 (sous presse).

FONTY G., GOUET Ph., SANTÉ V., 1988. Influence d'une bactérie méthanogène sur l'activité cellulolytique de deux espèces de champignons du rumen, in vitro. Résultats préliminaires. Reprod. Nutr. Dévelop., 28, 133-134.

FORD C.W., ELLIOTT R., MAYNARD P.J., 1987. The effect of chlorite delignification on digestibility of some grass forages and on intake and rumen microbial activity in sheep fed barley straw. J. agr. Sci. Camb., 108, 129-136.

GAY L., HEBRAUD M., GIRARD V., FEVRE M., 1988. La chitine synthase de Neocallimastix frontalis, un marqueur enzymatique de la biomasse fongique. Reprod. Nutr. Dévelop., 28, Suppl. $\mathrm{n}^{\circ} 1$.

GORDON G. L. R., 1985. The potential for manipulation of rumen fungi. Rev. rura/ Sci., 6, $124-128$.

GORDON G. L. R., ASHES J.R., 1984. In vitro digestion of wheat straw by different rumen anaerobic fungi. Can. J. anim. Sci., 64 (suppl.), 156-157.

GORDON G. L. R., GULATI S. K., ASHER J. R., 1983. Influence of low sulfur straw on anaerobic fungal numbers in a sheep rumen. Proc. Nutr. Soc. Aust., 8, 188.

GREENWOOD Y., HALL F. J., ORPIN C. G., PATERSON I. W., 1983. Microbiology of see-weed digestion in Orkney sheep. J. Physiol., 343, $121 \mathrm{p}$.

GRENET E., BARRY P., 1987. Etude microscopique de la digestion des parois végétales des téguments de soja et de colza dans le rumen. Reprod. Nutr. Dévelop., 27, 246-248.

GRENET E., BARRY P., 1988. Colonization of thick-walled plant tissues by anaerobic fungi. Anim. Feed Sci. Technol., 19, 25-31.

GRENET E., BRETON A., FONTY G., BARRY P., REMOND B., 1988 . Influence du régime alimentaire sur la population fongique du rumen. Reprod. Nutr. Dévelop., 28, 127-128.

GRENET E., BRETON A., BARRY P., FONTY G., 1989. Rumen anaerobic fungi and plant substrats colonization as affected by diet composition. Anim. Feed Sci. Technol. (sous presse).

GRENET E., FONTY G., BARRY P., 1988. SEM study of the degradation of maize and lucerne stems in the rumen of gnotobiotic lambs harbouring only fungi as ceilulolytic micro-organisms. In J. V. NOLAN, R. A. LENG, D. I. DEMEYER. The role of protozoa and fungi in ruminant digestion. Penambule Books, Armidale N.S.W. 2351, Australia. Proceedings of an International seminar sponsored by the O.E.C.D. Cooperative Research Project on Food and Preservation, and the University of New England, held 26-29, September 1988 (sous presse).

HEATH I. B., BAUCHOP T., SKIPP R. A., 1983. Assignment of the rumen anaerobe Neocallimastix frontalis to the Spizellomycetales (chytridiomycetes) on the basis of its polyflagellate zoospore ultrastructure. Can. J. Botany, 61, 295-307.

HEBRAUD M., FĖVRE M., 1988a. Caractérisation des hydrolases sécrétées par les champignons anaérobies du rumen. Reprod. Nutr. Dévelop., 28, 131-132.

HEBRAUD M., FĖVRE M., 1988b. Characterization of glycosides and polysaccharides hydrolases secreted by the rumen anaerobic fungi Neocallimastix frontalis, Sphaeromonas communis, and Piromonas communis. J. gen. Microbiol., 134, 1123-1129.

HO Y. W., ABDULLAH H., JALALUDIN S., 1988. Penetrating structures of anaerobic rumen fungi in cattle and swamp buffalo. J. gen. Microbiol., 134, 177-181.

HUNGATE R. E., 1969. A roll tube method for cultivation of strict anaerobes. In J. R. NORRIS, D. W. GIBBONS, Methods and microbiology. Acad. Press, New York and London, 38, 117-132.

IANOTTI E. L., KAFKEWITZ D., WOLIN M. S., BRYANT M. P., 1973. Glucose fermentation products of Ruminococcus albus grown in continuous culture with Vibrio succinogenes: changes caused by interspecies transfer of $\mathrm{H}_{2}$. J. Bact., 114, $1231-1240$. 
JOBLIN K. N., 1981. Isolation, enumeration and maintenance of rumen anaerobic fungi in roll tubes. Appl. Environ. Microbiol., 42, 1119-1122.

KEMP P., LANDER D. J., ORPIN C. G., 1984. The lipids of the rumen fungus Piromonas communis. J. gen. Microbiol, 130, 27-37.

KEMP P., JORDAN D. J., ORPIN C. G., 1985. The free and protein amino acids of the rumen phycomycetes fungi Neocallimastix frontalis and Piromonas communis. J. agric. Sci. Camb., 105, 523-526.

LIEBETANTZ, 1910. Die parasitischen Protozoen des Widerkauermagens. Arch. Protiskenk., 19, 19.

LOWE S. E., THEODOROU M. K., TRINCI A. P. J., HESPELL R. B., 1985. Growth of anaerobic rumen fungi on defined and semi-defined media lacking rumen fluid. J. Gen. Microbiol., 131, 2225-2229.

LOWE S. E., GRIFFITH C. G., MILNE A., THEODOROU M. K., TRINCI A. P. J., 1987a. The life cycle and growth kinetics of an anaerobic rumen fungus. J. gen. Microbiol., 133, 1815-1827.

LOWE S. E., THEODOROU M. K., TRINCI A. P. S., 1987b. Cellulases and xylanase of an anaerobic rumen fungus grown on wheat-straw lignocellulose, wheat-straw holocellulose, cellulose and xylan. Appl. environ. Microbiol., 53, 1216-1223.

LOWE S. E., THEODOROU M. K., TRINCI A. P. J., 1987c. Growth and fermentation of an anaerobic rumen fungus on various carbon sources and the effects of temperature on development. Appl. Environ. Microbiol., 53, 1210-1215.

LOWE S. E., THEODOROU M. K., TRINCI A. P. J., 1987d. Isolation of anaerobic fungi from saliva and faeces of sheep. J. gen. Microbiol., 133, 1824-1834.

MOUNTFORT D. O., 1987. The rumen anaerobic fungi. F.E.M.S. Microbiol. Rev., 46, $401-408$.

MOUNTFORT D. O., ASHER R. A., 1983. Role of catabolite regulatory mechanisms in control of carbohydrate utilization by the rumen anaerobic fungus Neocallimastix frontalis. Appl. environ. Microbiol., 46, $1331-1338$.

MOUNTFORT D. O., ASHER R. A., 1985. Production and regulation of cellulase by two strains of the rumen anaerobic fungus Neocallimastix frontalis. Appl. environ. Microbiol., 49, 1314-1322.

MUNN E. A., ORPIN C. G., HALL F. J., 1981. U/trastructural studies of the free zoospores of the rumen Phycomycete Neocallimastix frontalis. J. gen. Microbiol., 125, 311-323.

ORPIN C. G., 1975. Studies in the rumen flagellate Neocallimastix frontalis. J. gen. Microbiol., 91, 249-262.

ORPIN C. G., 1976. Studies on the rumen flagellate Sphaeromonas communis. J. gen. Microbiol., 94, 270-280.

ORPIN C. G., 1977a. The rumen flagellate Piromonas communis: its life-history and invasion of plant material in rumen. J. gen. Microbiol., 99, 107-117.

ORPIN C.G., 1977b. The occurrence of chitin in the cell walls of the rumen organisms Neocallimastix frontalis, Piromonas communis and Sphaeromonas communis. J. gen. Microbiol., 99, 215-218.

ORPIN C. G., 1977c. On the induction of zoosporogenesis in the rumen phycomycetes Neocallimastix frontalis, Piromonas communis and Sphaeromonas communis. J. gen. Microbiol. 101, $181-189$.

ORPIN C. G., 1981 a. Isolation of cellulolytic phycomycetes fungi from the caecum of the horse. $J$. gen. Microbiol., 123, 287-296.

ORPIN C. G., 1981b. Degradation of plant cell wall material. Agric. Sci. Semin., 34-46.

ORPIN C. G., 1984. The role of ciliate protozoa and fungi in the rumen digestion of plant cell walls. Anim. Feed Sci. Technol., 10, 121-143.

ORPIN C. G., BOUNTIFF L., 1978 . Zoospore chemotaxis in the rumen phycomycete Neocallimastix frontalis. J. gen. Microbiol., 104, 113-122.

ORPIN C. G., HART Y., 1980. Digestion of plant particles by rumen phycomycetes fungi. J. appl. Bact., 49, X.

ORPIN C. G., GREENWOOD Y., 1986a. The role of haems and related compounds in the nutrition and zoosporogenesis of the rumen chytridiomycete Neocallimastix frontalis H8. J. gen. Microbiol., 132, 2179-2185.

ORPIN C. G., GREENWOOD Y., 1986b. Nutritional and germination requirements of the rumen chytridiomycete Neocallimastix patriciarum. Trans. Brit. Mycol. Soc., 86, 103-109. 
ORPIN C. G., LETCHER A. J., 1979. Utilization of cellulose, starch, xylan, and other hemicelluloses for growth by the rumen phycomycete Neocallimastix frontalis. Curr. Microbiol., 3, 121-124.

ORPIN C. G., MUNN E. A., 1986. Neocallimastix patriciarum sp. nov, a new member of the Neocallimasticaceae inhabiting the rumen of sheep. Trans. brit. Mycol. Soc., 86, 178-181.

PEARCE P. D., BAUCHOP T., 1985. Glycosidases of the rumen anaerobic fungus Neocallimastix frontalis grown on cellulosic substrates. Appl, environ. Microbiol., 49, 1265-1269.

STEWART C. S., McPHERSON C. A., CANSUMAR E., 1987. The effect of lasalocid on glucose uptake, hydrogen production and the solubilization of straw by the anaerobic rumen fungus Neocallimastix frontalis. Lett. appl. Microbiol., 5, 5-7.

TILLEY J. M. A., TERRY R. A., 1963. A two stage technique for the in vitro digestion of forage crops. J. Br. Grass/. Soc., 18, 104-111.

WALLACE R. J., JOBLIN K. N., 1985. Proteolytic activity of a rumen anaerobic fungus. F.E.M.S. Microbiol. Lett., 29, 19-25.

WALLACE R. J., MUNRO C. A., 1986. Influence of the rumen anaerobic fungus Neocallimastix frontalis on the proteolytic activity of a defined mixture of rumen bacteria growing on a solid substrate. Lett. appl. Microbiol., 3, 23-26.

WILLIAMS A. G., ORPIN C. G., 1987a. Polysaccharide-degrading enzymes formed by three species of anaerobic rumen fungi on a range of carbohydrate substrates. Can. J. Microbiol., 33. $418-426$.

WILLIAMS A. G., ORPIN C. G., 1987b. Glycoside hydrolase enzymes present in the zoospore and vegetative growth stages of the rumen fungi Neocallimastix patriciarum, Piromonas communis, and identified isolate, grown on a range of carbohydrates. Can. J. Microbial., 33 , $427-434$.

WILLIAMS A. G., STRACHAN N. H., 1984. The distribution of polysaccharide degrading enzymes in the bovine rumen digesta ecosystem. Curr. Microbiol., 10, 215-220

WINDHAM W. R., AKIN D. E., 1984. Rumen fungi and forage fiber degradation. Appl. Environ. Microbiol., 48, 473-476.

WOOD T. M., McCRAE S. I., WILSON C. A., BHAT K. M., GOW L. A., MANNING J. E., 1987. Aerobic and anaerobic fungal cellulase with special references to their mode of attack on cristalline cellulose. F.E.M.S. Symp. Biochemistry and genetics of cellulose degradation. Paris, p. 13.

WOOD T. M., WILSON C. A., MCCRAE S. I., JOBLIN K. N., 1986. A highly active extracellulase from the anaerobic rumen fungus Neocallimastix frontalis. Microbiol. Lett., 34, 37-40.

YARLETT N. C., HANN A. C., LLOYD D., WILLIAMS A., 1981. Hydrogenosomes in the rumen protozoon Dasytricha ruminantium Schuberg. Biochem. J., 200, 365-372.

YARLETT N., ORPIN C. G., MUNN E. A., YARLETT N. C., GREENWOOD C. A., 1986a. Hydrogenosomes in the rumen fungus Neocallimastix patriciarum. Biochem. J., 236, 729-739.

YARLETT N. C., YARLETT N., ORPIN C. G., LLOYD D., 1986b. Cryopreservation of the anaerobic rumen fungus Neocallimastix patriciarum. Lett. appl. Microbio/., 3, 1-3. 